\section{The retinal reference of the tilt aftereffect*}

\author{
MAX COLTHEART $\dagger$ and CHRISTOPHER M. COOPER \\ University of Waterloo, Waterloo, Ontario, Canada
}

Day and Wade (1969) proposed that visual "normalization" and the visual tilt aftereffect depend upon the gravitational orientation of test and inducing figures and that the retinal orientation of these figures is irrelevant. Their failure to distinguish between "normalization" and aftereffect is pointed out, and an analysis of their experiment suggested that it could not yield data which would unambiguously support either the gravitational or the retinal viewpoint. An experiment was reported in which a tilt aftereffect was found to occur under conditions where inducing and test figures could not vary in gravitational orientation. It was concluded that retinal orientation is a sufficient factor in the tilt aftereffect situation; whether it is a necessary factor or whether gravitational orientation is also sufficient remains to be determined.

The apparent orientation of a straight line or grating pattern, the test figure (TF), is altered if the observer has previously inspected another such stimulus with a different orientation, the inspection figure (IF). Prior inspection of the IF shifts the apparent orientation of the TF away from the IF orientation. This is the tilt aftereffect (AE). The usual definition of this $A E$ in fact refers only to a special case of the effect, namely, to the fact that a TF formerly judged to be vertical will, after inspection of a tilted IF, appear to be tilted in a direction opposite to that of the IF (Gibson \& Radner, 1937).

A second effect that has been observed in experiments on the tilt AE and whose relationship to the tilt $A E$ is at present unclear is "normalization" (Gibson \& Radner, 1937), also called "neutralization" (Taylor, 1962). During inspection, the orientation of an IF appears gradually to change. If the IF orientation is between vertical and $45 \mathrm{deg}$, the IF appears to become more vertical; if the IF orientation is between $45 \mathrm{deg}$ and horizontal, the IF appears to become more horizontal. No neutralization occurs with vertical, horizontal, or 45-deg IFs. The tilt AE, however, does occur with a vertical IF (Kohler \& Wallach, 1966; Templeton, Howard, \& Easting, 1965) and with 45-deg and horizontal IFs (unpublished data of M. M. Taylor).

Whether or not neutralization and the tilt AE are merely different aspects of the same underlying process (Gibson \& Radner, 1937) or are distinct processes (Taylor, 1962), the two effects are operationally distinguishable because they are

*This research was supported by Grant APA-0350 from the National Research Council of Canada. We thank $C$. Berry and N. J. Wade for valuable discussions.

+ Requests for reprints should be sent to Max Coltheart, Department of Psychology, University of Waterloo, Waterloo, Ontario, Canada. measured differently. Neutralization is measured by obtaining judgments of the orientation of a stimulus before and after prolonged observation of that stimulus. The tilt $\mathrm{AE}$ is measured by obtaining judgments of the orientation of a stimulus before and af ter prolonged inspection of a stimulus having a different orientation. In other words, neutralization is what is being measured in experiments where the IF and TF have the same orientation, whereas the tilt $\mathrm{AE}$ is what is being measured in experiments where the IF and TF have different orientations.

In almost all discussions of the tilt $A E$ or of neutralization, the term "orientation" is ambiguous. It is not stated whether what is meant is orientation with respect to a retinal meridian or orientation with respect to a gravitational meridian. For example, when it is stated that neutralization does not occur with figures oriented vertically, horizontally, or at $45 \mathrm{deg}$, does this statement refer to retinal orientation? Again, when it is stated that the tilt $\mathrm{AE}$ is measured in experiments in which the IF and TF differ in orientation, does this mean that the two figures must have different retinal orientations or different gravitational orientations?

This issue has been raised by Prentice and Beardslee (1950) and by Day and Wade (1969). Prentice and Beardslee had Ss tilt their heads $10 \mathrm{deg}$ and view a line tilted $10 \mathrm{deg}$ in the same direction. This line was, therefore, retinally vertical (aince ocular countertorsion was taken into account by using an afterimage to position the head) but gravitationally tilted. Neutralization occurred; Prentice and Beardslee therefore concluded that a departure of gravitational orientation from the key orientations of vertical, $45 \mathrm{deg}$, and horizontal was sufficient to produce neutralization. A question left unanswered is whether, in this orientation or to gravitational situation, gravitational orientation is a necessary factor (retinal orientation being irrelevant) or merely a sufficient factor (in which case neutralization might be also influenced by retinal orientation). Nevertheless, Prentice and Beardslee's findings are of great importance, since they conflict with any theory which attempts to explain neutralization solely in terms of events occurring within the visual system.

Day and Wade (1969) do not distinguish between neutralization (normalization) and the tilt $\mathrm{AE}$, as will be clear from the second sentence of their paper: "Normalization has been measured indirectly from the difference between judgments of the visual vertical before and after a period of inspection, an effect referred to as the visual tilt aftereffect." Nevertheless, since it is still a subject of theoretical controversy whether neutralization and the tilt $\mathbf{A E}$ are substantively or merely operationally different, this distinction has been kept in mind in the following analysis of Day and Wade's experiment.

In order to make this analysis as explicit as possible, it is necessary to define four hypotheses as follows:

GN: A necessary and sufficient condition for the occurrence of neutralization is that the IF orientation is neither 0,45 , or $90 \mathrm{deg}$ with respect to gravity.

RN: A necessary and sufficient condition for the occurrence of neutralization is that the IF orientation is neither 0,45 , or $90 \mathrm{deg}$ with respect to the vertical retinal meridian.

GA: A necessary and sufficient condition for the occurrence of a tilt $\mathrm{AE}$ is that the gravitational orientation of the IF differs by an appropriate amount from the gravitational orientation of the TF.

RA: A necessary and sufficient condition for the occurrence of a tilt $\mathrm{AE}$ is that the retinal orientation of the IF differs by an appropriate amount from the retinal orientation of the $\mathbf{T F}$.

The critical conditions in Day and Wade's experiment are Conditions III and IV. In Condition III an upright $S$ made four pretest settings of a bar to the vertical. He was then tilted $15 \mathrm{deg}$ clockwise and for 2 min inspected a gravitationally vertical bar. Then, with his body still tilted (N. J. Wade, personal communication), he made a single posttest setting to the gravitational vertical. The aftereffect was defined as the difference between the posttest setting and the mean of the pretest settings. Day and Wade argued that no effect should occur in this situation if gravitational orientation is the critical factor because the IF was gravitationally 
vertical, whereas an effect should occur if retinal orientation is the critical factor, because the IF was not retinally vertical. The pretest-posttest difference in this condition was insignificant in each of two experiments.

Let us analyze this condition from a gravitational point of view and then from a retinal point of view. Gravitationally speaking, the experiment consists of four pretest settings to the gravitational vertical, a 2-min inspection of a gravitationally vertical line, and then one posttest setting to the gravitational vertical. Obviously no difference would be expected between pretest and posttest here. Neutralization would not occur because the IF is vertical. The tilt $A E$ is not being measured here because the orientation to which the TF is to be set coincides with the IF orientation. Thus, this experiment is relevant to Hypothesis GN, which predicts no effect under these conditions.

Retinally speaking, the experiment may be described thus: (1) four pretest settings to the retinal vertical; (2) 2-min inspection of a line tilted 15 deg counterclockwise from the retinal vertical; (3) one posttest setting to $15 \mathrm{deg}$ counterclockwise from the retinal vertical. A tilt $A E$ will not occur here because the posttest setting of the TF will not be affected by the IF, since the orientation to which the TF is to be set coincides with the IF orientation. Neutralization of the IF ought to occur; but this paradigm is inappropriate for detecting it. The retinal orientation to which the TF had to be set in the pretest (the retinal vertical) was retinally different from the retinal orientation to which the TF had to be set in the posttest (15 deg counterclockwise from the retinal vertical). Quite apart from the unpredictable effects this might have had, which would be confounded with and hence could cloak the effects of neutralization, there is a quite specific and predictable confounding effect, namely, proprioceptive adaptation (Day \& Wade, 1966; Wade, 1968; Wade \& Day, 1968a, b). After a period of clockwise head tilt with eyes shut, the apparent visual vertical is displaced clockwise. Since, in Condition III, retinal neutralization would produce a counterclockwise shift of the apparent vertical, retinal neutralization would be concealed by the effects of proprioceptive adaptation in this condition. The insignificance of the pretest-posttest difference is thus uninterpretable. The picture is still further complicated by a second factor, the $E$ effect; judgments of the visual vertical are affected by whether or not the head is tilted during judgment (Wade, 1969), and this too is confounded with the neutralization effect, since it would contribute to the pretest-posttest comparison.

Consequently, while the negative results of Condition III are consistent with the gravitational viewpoint, the absence of a significant difference between pretest and posttest cannot be taken as evidence against the retinal viewpoint because of the confounding effects introduced by the difference in head tilt between pretest and posttest and by the effects of proprioceptive adaptation. If these confounding factors had not been present, the experiment would have been relevant to Hypothesis RN, which predicts a neutralization effect under these conditions; but, for the reasons given above, a legitimate test of $R N$ could not be achieved in Condition III.

Let us now turn to Condition IV of Day and Wade's experiment. Here an upright $\mathbf{S}$ made four pretest settings of a bar to the vertical. He was then tilted $15 \mathrm{deg}$ clockwise and, for $2 \mathrm{~min}$, inspected a bar also tilted $15 \mathrm{deg}$ clockwise (and therefore retinally vertical, neglecting the slight effects of ocular countertorsion). Then, with his body still tilted, he made a single posttest setting to the gravitational vertical. Day and Wade argued that an effect should occur in this situation if gravitational orientation is the critical factor, because the IF was gravitationally tilted; since the IF was retinally vertical, no effect should occur if the critical factor is retinal orientation. The pretest-posttest difference in this condition was significant in each of their two experiments.

The gravitational analysis of this condition is as follows: (1) four pretest settings to the gravitational vertical; (2) 2-min inspection of a line tilted $15 \mathrm{deg}$ clockwise from the gravitational vertical; (3) one posttest setting of the line to the gravitational vertical. $A$ tilt $A E$ would be expected here. Neutralization is not being measured because the IF orientation differs from the TF orientation. The hypothesis being tested is GA, which predicts that an effect will occur.

Retinally, this condition is as follows: (1) four pretest settings to the retinal vertical; (2) 2-min inspection of a line which is retinally vertical; (3) one posttest setting to an orientation $15 \mathrm{deg}$ counterclockwise of the retinal vertical. No neutralization would be expected here because the IF is retinally vertical. $A$ tilt AE would be expected because the IF is $15 \mathrm{deg}$ away from the orientation to which the TF is to be set. Thus, this condition tests Hypothesis RA, which predicts that an effect will occur.

Now, Day and Wade argued that a tilt AE would not be predicted here from retinal considerations because the IF is retinally vertical. Presumably their reason for so arguing was the belief that IFs coinciding with the "norms of visual space" (the vertical and the horizontal) do not generate tilt AEs, a view implied by the normalization theory of tilt AEs (Gibson \& Radner, 1937). This belief is, however, incorrect. Retinally vertical IFs do produce tilt AEs when the orientation to which the TF is to be set is not the retinal vertical (Kohler \& Wallach, 1944, pp. 309-311; Templeton, Howard, \& Easting, 1965).

Thus, in Condition IV, if gravitational orientation is critical, a tilt AE, manifested as a significant difference between pretest and posttest settings, should occur. If retinal orientation is the critical factor, the same thing should happen. Therefore, the observed significant difference between pretest and posttest settings in this condition does not discriminate between the retinal and gravitational viewpoints.

This conclusion assumes that the significant difference between pretest and posttest in Condition IV is a genuine aftereffect, but even this assumption could be challenged. First, as mentioned above, the effects of proprioceptive adaptation would be sufficient to produce a difference between pretest and posttest; its effect would be to make the posttest settings clockwise of the pretest settings. Second, the E effect would differentially influence pretest and posttest settings, making the posttest settings counterclockwise of the pretest settings.

The general conclusion from this detailed examination of Day and Wade's experiment is as follows: It is true that neutralization should have occurred in their Condition III if it is a retinal effect, but the difference in body-tilt conditions between pretest and posttest conditions, plus the confounding effects of proprioceptive adaptation and of the $E$ effect, do not allow any conclusions to be drawn from the lack of an effect in this condition. With respect to Condition IV, it is incorrect to argue that the retinal viewpoint predicts that no effect should occur; both the retinal and gravitational viewpoints predict an effect, and the obtained effect, insofar as we are willing to ignore the confounding effects of proprioceptive adaptation and the $E$ effect, is consistent with both viewpoints.

The experiment we report here is a first step in an attempt to clarify these issues. We are concerned merely with whether a retinal difference between IF and TF in the absence of any gravitational difference is sufficient to 
produce a tilt AE. Insofar as Day and Wade identified the tilt $\mathrm{AE}$ and neutralization, they denied that a retinal difference in the absence of a gravitational difference would produce a tilt AE. One situation in which this question could be investigated is under conditions of zero gravity, as in space flight. Another such condition involves a $S$ lying on the floor and making judgments of the orientation of a line on the ceiling. We chose to use the latter situation.

\section{APPARATUS AND PROCEDURE}

$A$ bar $24 \mathrm{in}$. long and $7 / 8 \mathrm{in}$. in diam was suspended from the ceiling of a light-tight dark room. The bar was horizontal and was painted with fluorescent green paint so that it was visible when illuminated by a black light. The floor and walls of the room were painted flat black and the ceiling covered with matte black cloth.

A collapsible bed was located below the bar so that each $S$ could lie with the midpoint of his eyes directly beneath the center of the bar. A plumb line was used to position S's head correctly. The distance from head to bar was 78 in. The center of the bar was covered with a small piece of black tape (the fixation point).

The bar could be rotated about its center in a horizontal plane by means of a 1-rpm synchronous reversible motor. This rotation was under the control of the $S$, who held a pressbutton in each hand. Depression of one button produced clockwise rotation, and depression of the other button produced anticlockwise rotation. A clutch mechanism between bar and motor ensured that the bar stopped moving immediately when a button was released.

The orientation of the bar was displayed on a meter visible to $\mathrm{E}$, who was also equipped with a switch which controlled the bar orientation.

Eleven undergraduates at the University of Waterloo served as Ss. After the $S$ was positioned on the bed, the operation of the hand controls was explained to him and he was instructed to fixate the fixation point at all times. He was then asked to align the bar to his body axis by using the hand controls. Ten such trials were run, five using a starting point $27 \mathrm{deg}$ clockwise from the body axis and five $30 \mathrm{deg}$ counterclockwise from the body axis, in a random order. The mean of these 10 settings will be referred to as the subjective body axis (SBA).

Two IF orientations (20 deg clockwise and counterclock wise from the SBA) were used, and with each IF orientation, two starting positions ( 6 deg clockwise and counterclock wise from the SBA) were used. The tilt AE
Table 1 Mean AE for Each S*

\begin{tabular}{cc}
\hline$S$ & AE in Degrees \\
\hline 1 & +2.40 \\
2 & +0.30 \\
3 & +1.87 \\
4 & +2.25 \\
5 & +0.98 \\
6 & +1.35 \\
7 & +0.90 \\
8 & +1.28 \\
9 & +1.62 \\
10 & +4.12 \\
11 & +1.42 \\
Mean & +1.68 \\
\hline
\end{tabular}

*In calculating these values, an $A E$ in the expected direction was always given a + sign.

was measured twice under each of these four possible conditions, the eight measurements being made in a random order.

Each measurement began with a pretest in which the bar was oriented at the appropriate starting position and the $S$ instructed to align the bar to his body axis. Only one reversal of the direction of rotation of the bar was permitted during each setting of the bar by Ss. After this pretest setting, $S$ was told to close his eyes, the bar was oriented at the appropriate IF orientation, and $\mathrm{S}$ was told to open his eyes and fixate the center of the bar. After a 2-min inspection period, the $\mathrm{S}$ was told to close his eyes, the bar was returned to the starting position used in the pretest, and $S$ was told to open his eyes and align the bar to his body axis, again with only one reversal of the direction of rotation permitted. The AE was defined as the difference between the pretest and posttest settings. A 1-min interval, during which S's eyes were closed, elapsed between each posttest and the next pretest to permit dissipation of the AE.

\section{RESULTS AND DISCUSSION}

Table 1 shows the mean AE for each of the $11 \mathrm{Ss}$, a positive sign denoting an $\mathrm{AE}$ in the expected direction (posttest setting closer than pretest setting to IF orientation). Every $S$ produced a mean $A E$ in the expected direction.

These data indicate that an appropriate difference in the retinal orientations of IF and TF, with constant gravitational orientation, is sufficient for the production of a tilt AE. The data are thus inconsistent with the conclusion of Day and Wade (1969) that it is gravitational orientation rather than retinal orientation which is of critical importance in this situation. The data thus support Hypothesis RA and are inconsistent with Hypothesis GA. Weaker forms of Hypotheses GA, RN, and GN remain to be tested, and thus the contribution of the present experiment to our knowledge of the roles played by gravitational and retinal orientation in the tilt aftereffect and neutralization situations is rather modest. Nevertheless, the rejection of the strong claim that only gravitational factors are important is of some significance.

It has been proposed (Coltheart, 1971) that the tilt $A E$ is attributable to the adaptation of orientation-specific units in the visual cortex (Hubel \& Wiesel, 1959). Since these units are, in general, specific to retinal orientation, this theory is embarrassed by the claim that the tilt AE depends upon gravitational orientation. Perhaps (Coltheart, 1971) the occurrence of a tilt $\mathrm{AE}$ relative to gravitational orientation might be related to the finding by Horn and Hill (1969) and Spinelli (1970) that some orientation-specific units can compensate for head tilt and thus can respond to gravitational orientation. The difficulty with this attempt to cope with Day and Wade's results is that it takes many minutes for these units to adjust to a new body tilt, and this does not fit well with Day and Wade's data, which appeared to imply extremely rapid compensation for body tilt. A more serious difficulty for any explanation of the tilt AE in terms of adaptation of orientation-specific units is that the units which compensate for head tilt are far less numerous than those which do not. The latter units would produce a tilt AE which depended on retinal orientation, and Day and Wade claimed that no such AE exists. No solution to this problem was offered by Coltheart (1971).

Our findings dispose of these difficulties for the theory that the tilt AE occurs because of the adaptation of orientation-specific units, since it now seems to be the case that our findings and those of Day and Wade indicate that an appropriate difference in retinal orientation of IF and TF is sufficient for the production of a tilt $A E$, which is what is required by the adaptation theory of the tilt $\mathrm{AE}$, but which was denied by Day and Wade. Whether a retinal difference between IF and TF is necessary as well as sufficient is not known; to put this another way, whether a gravitational difference between IF and TF is sufficient is unknown. This can be decided by repeating Day and Wade's experiment with all Ss upright during pretest and posttest but tilted during the inspection phase. If a line tilted so as to be retinally vertical during the inspection phase influences posttest settings to the gravitational vertical during an upright posttest, this will be evidence for a gravitational 
determinant of the tilt $A E$. (Proprioceptive adaptation can be controlled for by using control Ss with shut eyes during the inspection phase.) A gravitational effect may well occur, since Prentice and Beardslee's (1950) results do seem to require that gravitational orientation can be a factor in neutralization. If a gravitational tilt AE occurs, any attempts to explian the tilt $\mathrm{AE}$ in terms of the adaptation of orientation-specific units will need to implicate both retinally specific and gravitationally specific units.

\section{REFERENCES}

COLTHEART, M. Visual feature-analyzers and aftereffects of tilt and curvature. Psychological Review, 1971, 78, 114-121.

DAY, R. H., \& WADE, N. J. Visual spatial aftereffect from prolonged head tilt. Science, 1966, 154, 1201-1202.
DAY, R. H., \& WADE, N. J. The reference for visual normalization. American Journal of Psychology, 1969, 82, 191-197.

GIBSON, J. J.. \& RADNER, M. Adaptation, aftereffect and contrast in the perception of tilted lines: I. Quantitative studies. Journal of Experimental Psychology, $1937,20,453-467$.

HORN, G., \& HILL, R. M. Modifications of receptive fields in the visual cortex occurring spontaneously and associated with bodily tilt. Nature, 1969, 221, 186-188.

HUBEL, D. H., W WESEL, T. N. Receptive fields of single neurones in the cat's striate cortex. Journal of Physiology. $1959,148,574-591$.

KOHLER, W., \& WALLACH, H. Figural aftereffects: An investigation of visual processes. Proceedings of the American Philosophical Society, 1944, 88, 269-357.

PRENTICE, W. C. H.. \& BEARDSLEE, D. C. Visual "normalization" near the vertical and horizontal. Journal of Experimental Psychology, 1950, 40, 355-364.

SPINELLI, D. N. Recognition of visual patterns. In D. A. Hamburg (Ed.), Perception and its disorders. Baltimore: Williams \& Wilkins, 1970.

TAYLOR, M. M. Figural aftereffects: A theory. Canadian Joumal of Psychology, 1962, 16, 247-277.

TEMPLETON, W. B., HOWARD, I, P \& EASTING, G. Satiation and the tilt aftereffect. American Joumal of Psychology, 1965, 7 , 656-659.

WADE, N. J. Visual orientation during and after lateral head, body, and trunk tilt. Perception \& Psychophy sics, 1968, 3, 215-219.

WADE, N. J. Visual orientation as a function of head tilt. Perceptual \& Motor Skills, 1969, 29, 573-574.

WADE, N. J., \& DAY, R. H. Development and dissipation of a visual spatial aftereffect from prolonged head tilt. Journal of Experimental Psychology, $1968 a, 76,439-443$.

WADE, N. J DAY, R. H. Apparent head position as a basis for a visual aftereffect of prolonged head tilt. Perception \& Psy chophysics, 1968b, 3, 324-326. (Accepted for publication December 2, 